\title{
Análisis de los beneficios de la actividad física en situaciones de crisis en jóvenes universitarios con síntomas depresivos
}

\author{
John D. Hoyos-Cifuentes ${ }^{1 *}$ y César A. Bernal-Torres ${ }^{2}$ \\ (1) Facultad de educación, Corporación universitaria minuto de Dios - UNIMINUTO Calle 1, numero 9 - 50, Bogotá D.C. \\ Colombia (correo-e: jhoyoscifue@uniminuto.edu.co) \\ (2) Escuela Internacional de Ciencias Económicas y Administrativas, Universidad de La Sabana, Km. 7, Autopista Norte \\ de Bogotá, Puente del Común, Campus Universitario, Chía, Colombia. (correo-e: cesar.bernal@unisabana.edu.co)
}

Recibido May. 24, 2021; Aceptado Jul. 21, 2021; Versión final Ago. 26, 2021, Publicado Dic. 2021

\begin{abstract}
Resumen
El presente estudio se enfocó en analizar los beneficios de la actividad física en jóvenes universitarios con síntomas depresivos. La investigación se realizó con 30 participantes no necesariamente diagnosticados clínicamente como depresivos. Se utilizó el inventario de depresión de Beck (BDI-II) antes y después de un programa de intervención basado en la realización de diferentes ejercicios físicos para determinar su incidencia en los estados de depresión. Para el análisis de los datos, se utilizó la prueba de los rangos con Signo de Wilcoxon. Los resultados indicaron que si hubo diferencia significativa entre el nivel de síntomas de depresión antes del programa de actividad física y después del programa, evidenciado una reducción significativa en síntomas depresivos. Se concluye que un buen programa de actividad física realizado de manera moderada presenta beneficios en la reducción de los niveles de depresión en jóvenes universitarios debido a que permite generar espacios de bienestar y mejoramiento de la calidad de vida.
\end{abstract}

\section{Analysis of physical activity benefits in crisis situations for university students with depressive symptoms}

\begin{abstract}
The present study focused on analyzing the benefits of physical activity in college students with depressive symptoms. The research was conducted with 30 participants that were not necessarily clinically diagnosed as depressive. The Beck depression inventory (BDI-II) was applied before and after an intervention program. Assessments were based on student performance in different physical exercises to determine their impact on depression. Data was analyzed by using the Wilcoxon signed-rank test. The results indicate that there was a significant difference between depression symptom levels before and after a physical activity, thereby showing a significant reduction in depressive symptoms. In conclusion, well-designed physical activity programs, performed in moderation, beneficially reduce depression levels in young university students since they generate positive spaces for improving student well-being and quality of life.
\end{abstract}

Keywords: physical activity; benefits; depression; youth; depressive symptoms 


\section{INTRODUCCIÓN}

Los hallazgos de diversas investigaciones acerca de los factores comportamentales que aquejan a las sociedades de hoy día, evidencian que la depresión constituye una de las causas de mayor riesgo para la salud mental. Esta aqueja a más de 300 millones de personas a nivel mundial (Ruggieri, 2020). La depresión en la actualidad crece significativamente alertando a los organismos gubernamentales que tienen la responsabilidad de combatir estos flagelos, como el fin de garantizar estados de vida saludables para la población en general. Una aproximación a la problemática planteada por la organización mundial de la salud (OMS) para quienes la depresión es: "un trastorno mental frecuente, que se caracteriza por la presencia de tristeza, pérdida de interés o placer, sentimientos de culpa o falta de autoestima, trastornos del sueño o del apetito, sensación de cansancio y falta de concentración" (Martínez, 2018).

Según este concepto, dicho estado puede generar dificultades en el desempeño del ser humano en su ámbito familiar, laboral y social y se espera que la depresión sea la causa de mayor incapacidad para el 2030 (Barceló, 2019). Una de las causas que más aquejan a la población con síntomas podría deberse a que en la actualidad se van generando nuevas formas de interconectividad como, por ejemplo, el uso de la computadora en el aumento del riesgo de depresión (Khouja, 2019). Para Disease (2015), la depresión es el trastorno más común en los jóvenes. No obstante, esta alteración del comportamiento es poco detectable. La idea es lograr más tratamientos para su reducción en términos cognitivos, emocionales, de regulación y desarrollar estrategias de afrontamiento. Esta alteración del comportamiento que contribuye a la discapacidad a lo largo de la vida se inicia en la juventud, además requiere de atención especializada (Davey y McGorry, 2019).

Diversos estudios demuestran la relación bidireccional entre la actividad física y la salud mental de los jóvenes. Es importante crear programas activos y alcanzables para mejorar la salud psicológica (Pascoe y Parker, 2019). Según McCrorie et al., (2014), las características ambientales tienen influencias significativas en los niveles de actividad física de los jóvenes. Para Kavanaugh et al., (2015), los programas de actividad física deben buscar ser agradables para los jóvenes por su relación objetiva y subjetiva con estos. Los programas basados en ejercicio físico y actividad física reducen las posibilidades de padecer algún tipo de alteración que comprometa la salud mental de la población. Su promoción es una prioridad para los organismos de salud. Esta es una razón para el aumento aceptable de programas de este tipo en personas de diferentes edades y grupos sociales, países, comunidades y especialmente de la población juvenil (Heath et al., 2012).

Para Whitelaw et al. (2010), la actividad física generalmente mejora los niveles de bienestar mental de los jóvenes. Así mismo, la actividad física y los diferentes programas de ejercicio y prácticas de yoga, stretching, etc., es decir, ejercicios centrados en la autoconciencia, se presentan como una oportunidad favorable para orientar a los jóvenes para que por medio de estas prácticas disminuyan la posibilidad de caer en estados depresivos y de otras patologías. Además, los ejercicios físicos contribuyen con el autocontrol, como una habilidad que facilita la autorregulación de conductas, emociones y pensamientos saludables (Pérez et al., 2018). Para Bailey et al. (2018), la actividad física aeróbica supervisada y con una intensidad de moderada a vigorosa, realizada tres veces por semana, durante algunas semanas, es una actividad que favorece la salud física y mental de los adolescentes y los adultos jóvenes con depresión.

Esta población particularmente demuestra una actitud de agrado hacia los diversos programas de actividad física existentes, pese a que su práctica disminuye con el paso de los años (Tannehill et al., 2015). No obstante, y teniendo en cuenta a Telama et al., (2005), la actividad física practicada en edad juvenil es significativa para su práctica en la vida adulta y esto representa una gran incidencia en la salud pública para la cual la actividad física se asocia con múltiples beneficios y mantiene buenos niveles de salud en la población juvenil, siendo esta una de las grandes preocupaciones de los organismos gubernamentales (Van Sluijs y Kriemler, 2016).

Según Parker et al. (2016), mediante la práctica de la actividad física se reducen los síntomas depresivos en jóvenes y estos programas pueden ser incluidos dentro de una intervención en la práctica clínica habitual. Bauman et al. (2012) aseguran que los distintos factores genéticos y sus diferentes características como la edad y el sexo, asociados a la motivación, la autoeficacia y el estado de salud entre otros, están en estrecha relación con el nivel de actividad física de una persona. Contrario a esto, los comportamientos sedentarios sobre todo en la juventud generalmente marcan el comportamiento futuro (Biddle et al., 2010). Lake y Townshend (2013) resaltan la importancia de la promoción de actividad física dentro de los comportamientos y estilos de vida en jóvenes, en tanto estos son significativos en el fomento de reducción de la obesidad, sobre todo en mujeres quienes al parecer son las que más prestan atención a asuntos relacionados con su peso (Zueck et al., 2015). Algunas enfermedades no trasmisibles se pueden prevenir con la creación y desarrollo de un buen programa de actividad física (Tikanmäki et al., 2017).

Por lo antes mencionado y teniendo en cuenta, que en la actualidad el distanciamiento social causado por la Covid 19, todas las personas nos hemos visto abocados a estar tiempos prolongados en casa y por ende a 
reducir las posibilidades de realizar ejercicio físico, pero más enfrentados a situaciones generadoras de depresión por la misma situación del Covid 19, este estudio se orientó a analizar en momentos de la pandemia los efectos de la actividad física en jóvenes con síntomas depresivos y con ello contribuir a resolver parte de este flagelo que está afectando a un número significativo de personas jóvenes y que para el casos de los universitarios tiene graves implicaciones psíquicas y académicas.

\section{METODOLOGÍA}

El estudio se realizó con un seguimiento longitudinal cuasi-experimental de diseño pre-post facto centrado en la aplicación de un plan de intervención, el cual se basó en un programa de actividad física que se desarrolló en un nivel de moderado a intenso de dos a tres veces por semana durante dos meses según lo descrito por Bailey et al. (2018). Cada sesión tuvo una duración de 40 minutos promedio, en la que se realizaron actividades de tipo aeróbico musicalizadas, zumba y taebo, ejercicios de fuerza tanto para el tren superior como para el inferior y ejercicios de yoga, taichí y stretching para generar espacios de relajación, autoconciencia y tranquilidad teniendo en cuenta que estos resultan ser benéficos para personas que presentan síntomas depresivos.

\section{Participantes}

La información del estudio se obtuvo de un total de 30 estudiantes de una universidad privada en Colombia (con mayoría de estudiantes de estratos socio económico medio y bajo). Para la vinculación de los estudiantes a la investigación se hizo una invitación formal, a través del área de bienestar universitario de la universidad enviada a todos los estudiantes de las sedes de Bogotá y Bello, donde se les presentaba los objetivos del estudio y se aclaraba que la invitación se orientaba específicamente a estudiantes (de cualquier carrera o semestre académico) que durante los últimos meses según su propia percepción hubieran sentido síntomas de depresión y que no estuvieran realizando ejercicios físicos de forma regular (diaria).

En total, 56 estudiantes respondieron a la invitación y se les informó de las actividades a realizar durante su participación voluntaria y de la reserva absoluta de la información personal y del uso de los resultados de la investigación solo para propósitos académicos. También, se les presentó el consentimiento informado para su firma. De ese total, 43 estudiantes integraron el grupo de participantes del proyecto, pero, solo 30 fueron los que se permanecieron durante todo el proceso.

\section{Instrumento - Inventario de depresión de Beck}

Para la obtención de la información relacionada con el estado de depresión en los estudiantes se utilizó el inventario de depresión de Beck, también conocido internacionalmente como test de Beck o BDI-II. Esta es una de las pruebas estandarizados más utilizadas con características psicométricas adecuadas para medir y cuantificar la gravedad de los niveles de intensidad depresivos en muestras clínicas o no clínicas de sujetos con síntomas depresivos sin necesidad de ser diagnosticados clínicamente. El test evalúa la sintomatología de la depresión con 21 ítems cada uno con cuatro posibilidades de respuesta (Sanz \& García 2013; Sanz et al., 2014). Para efectos de este estudio se utilizó la escala Likert de 1 a 4.

Vale aclarar que, para el desarrollo del proyecto, no se consideró necesario la aprobación del mismo por parte de un comité ético, debido a que, el programa de actividad física por ser de un nivel básico en donde los ejercicios no representaban ningún riesgo físico o mental para los participantes quienes todos fueron mayores de edad y participaron de manera voluntaria con conocimiento previo de los objetivos del estudio y de los ejercicios a realizar, así como la finalidad de la información obtenida.

\section{Procedimiento}

El desarrollo del estudio se inició con la aplicación del inventario de depresión o test de Beck que se orienta a avaluar el cómo se ha sentido la persona en la última semana previa e incluido el día de su aplicación. Luego se procedió a la realización del programa de intervención que tuvo una duración de dos meses, se desarrollaron dieciséis sesiones de actividad física entre las que se encontraron sesiones de stretching, musicalizada aeróbica, taichí, entrenamiento de fuerza del tren inferior y superior, zumba, yoga y taebo. Todas con una duración de aproximadamente 40 minutos a una intensidad moderada, es decir entre el $60 \%$ y el $70 \%$ de la frecuencia cardiaca máxima (FCM).

Las sesiones fueron organizadas estratégicamente alternando una de prevalencia aeróbica como zumba, musicalizada aeróbica o taebo, con una de relajación y autoconciencia como taichí, stretching y yoga con la intensión de que los participantes encontraran una gran variedad de técnicas y les resultara agradable el programa de intervención el cual es desarrollado por el profesional en ciencias del deporte y la educación física, entrenador físico y master en educación quien además es uno de los investigadores del presente 
estudio. La Tabla 1 denominada programa de actividad física, ilustra sobre la aplicación de las diferentes sesiones de actividad física realizadas durante el estudio.

Tabla 1. Programa de actividad física. FCM: Frecuencia Cardiaca Máxima. N/A: No aplica

\begin{tabular}{|c|c|c|c|c|c|}
\hline Dia & Actividad & Tipo de actividad & Repeticiones & Intensidad & Duración \\
\hline 1 & Strertching & Autoconciencia & $\begin{array}{l}20 \text { segundos } \\
\text { por musculo }\end{array}$ & Baja & 40 minutos \\
\hline 2 & Clase Musicalizada & Aeróbica & $\mathrm{N} / \mathrm{A}$ & Moderada 60 a $70 \%$ FCM & 40 minutos \\
\hline 3 & Taichi & Autoconciencia & $\mathrm{N} / \mathrm{A}$ & Moderada 60 a $70 \%$ FCM & 40 minutos \\
\hline 4 & Fuerza tren inferior & Fuerza aeróbica & $\begin{array}{l}12 \text { a } 15 \\
\text { repeticiones } \\
\text { por musculo }\end{array}$ & $\mathrm{N} / \mathrm{A}$ & 40 minutos \\
\hline 5 & Zumba & Aeróbica & $\mathrm{N} / \mathrm{A}$ & Moderada 60 a $70 \%$ FCM & 40 minutos \\
\hline 6 & yoga & Autoconciencia & $\mathrm{N} / \mathrm{A}$ & Baja & 40 minutos \\
\hline 7 & Taebo & Aeróbica & $\mathrm{N} / \mathrm{A}$ & Moderada 60 a $70 \%$ FCM & 40 minutos \\
\hline 8 & Clase Musicalizada & Aeróbica & $\mathrm{N} / \mathrm{A}$ & Moderada 60 a $70 \%$ FCM & 40 minutos \\
\hline 9 & Strertching & Autoconciencia & $\begin{array}{l}20 \text { segundos } \\
\text { por musculo }\end{array}$ & Baja & 40 minutos \\
\hline 10 & $\begin{array}{l}\text { Fuerza tren } \\
\text { superior }\end{array}$ & Fuerza aeróbica & $\begin{array}{l}12 \text { a } 15 \\
\text { repeticiones } \\
\text { por musculo }\end{array}$ & $\mathrm{N} / \mathrm{A}$ & 40 minutos \\
\hline 11 & Yoga & Autoconciencia & $N / A$ & Baja & 40 minutos \\
\hline 12 & Taebo & Aeróbica & $N / A$ & Moderada 60 a $70 \%$ FCM & 40 minutos \\
\hline 13 & Stretching & Autoconciencia & $\begin{array}{l}20 \text { segundos } \\
\text { por musculo }\end{array}$ & Baja & 40 minutos \\
\hline 14 & Zumba & Aeróbica & $\mathrm{N} / \mathrm{A}$ & Moderada 60 a $70 \%$ FCM & 40 minutos \\
\hline 15 & Clase Musicalizada & Aeróbica & $\mathrm{N} / \mathrm{A}$ & Moderada 60 a $70 \%$ FCM & 40 minutos \\
\hline 16 & Fuerza tren inferior & Fuerza aeróbica & $\begin{array}{l}12 \text { a } 15 \\
\text { repeticiones } \\
\text { por musculo }\end{array}$ & $\mathrm{N} / \mathrm{A}$ & 40 minutos \\
\hline
\end{tabular}

Por motivos del distanciamiento físico, las sesiones de actividad física se realizaron vía virtual mediante las diferentes plataformas de Google y Microsoft existentes para reuniones virtuales. Una vez terminado el plan de intervención basado en un programa de actividad física, se procedió a la aplicación del segundo inventario de depresión o cuestionarios de Beck, con el cual se buscó dar continuidad al objetivo de la investigación. Con la segunda aplicación se pretendió establecer o identificar los cambios que los participantes pudieron presentar una vez realizado el programa de actividad fisca.

\section{RESULTADOS}

Con relación a los datos sociodemográficos de los estudiantes participantes del estudio, se encontró que, 17 fueron mujeres y 13 hombres, la edad estuvo entre 19 y 42 años; 19 eran soltero(a)s, 6 casados, 4 vivían en unión libre y 1 separado; 14 tenían un hijo, 12 no tenían hijos, 4 tenía dos hijos y 1 tenía tres hijos; 20 se encontraban laborando y 10 solo dedicados al estudio; 17 desempeñaban rol cabeza de hogar.

De acuerdo con la escala de puntuaciones de 1 a 4 para los 21 ítems evaluados, los resultados obtenidos en cuanto a nivel de síntomas de depresión muestran que, en rango normal (bajo nivel de depresión) en la evaluación inicial del BDII se ubicaron el $40 \%$ de los participantes, mientras que, en ese mismo rango en la evaluación final se ubicaron el $73,3 \%$ de ellos. Por género, en ese rango normal se ubicó el $41,2 \%$ de las mujeres y el $38 ; 5 \%$ de los hombres, mientras que, una vez realizada la actividad física en ese rango se ubicaron el $64,7 \%$ de las mujeres y el $84,6 \%$ de los hombres (Figura 1 ). 

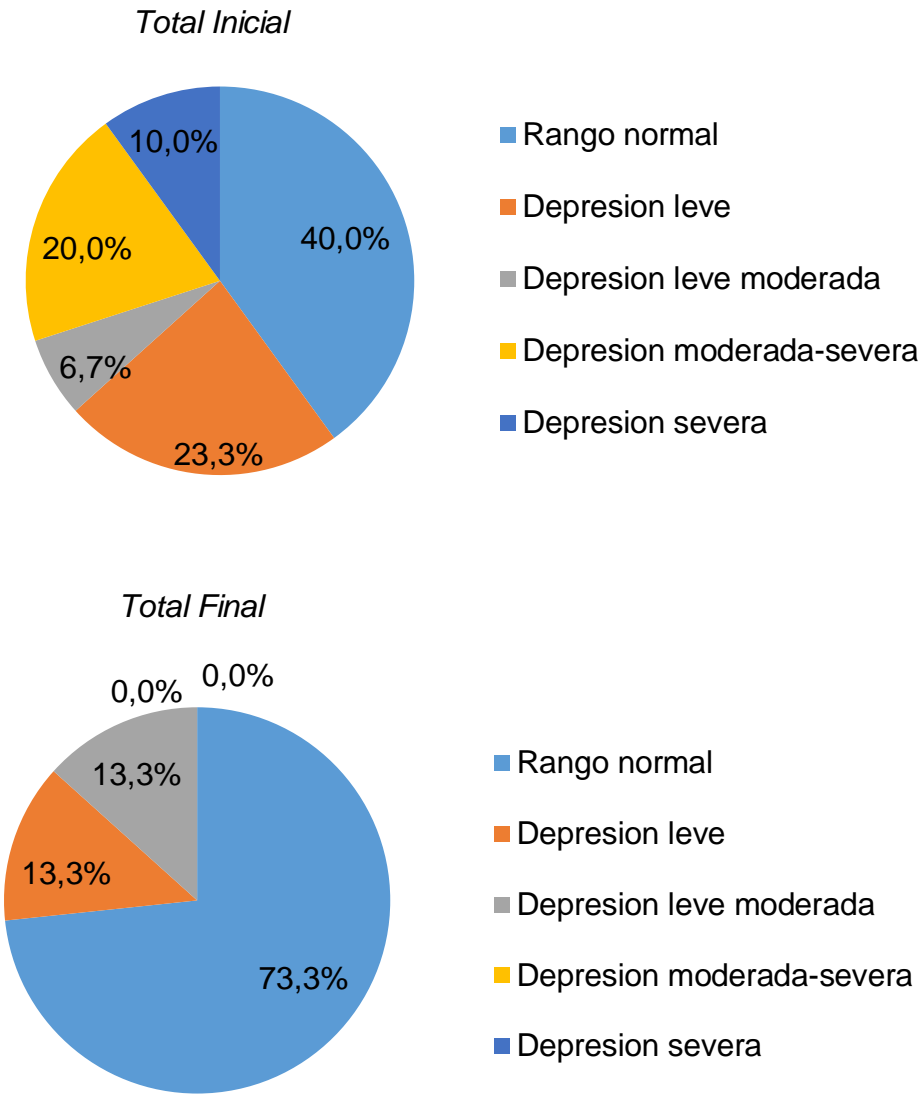

Fig. 1. Nivel de síntomas de depresión al inicio y al final del programa de actividad física para el conjunto de los participantes

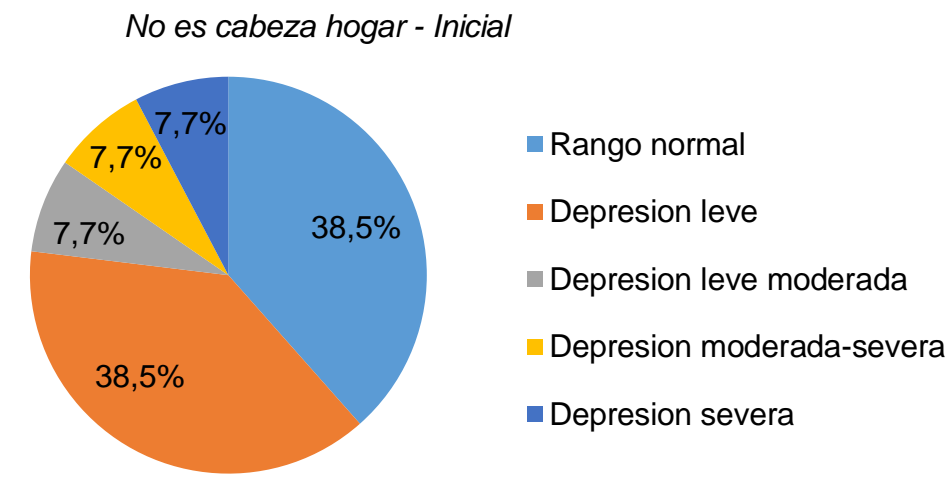

No es cabeza hogar - Final

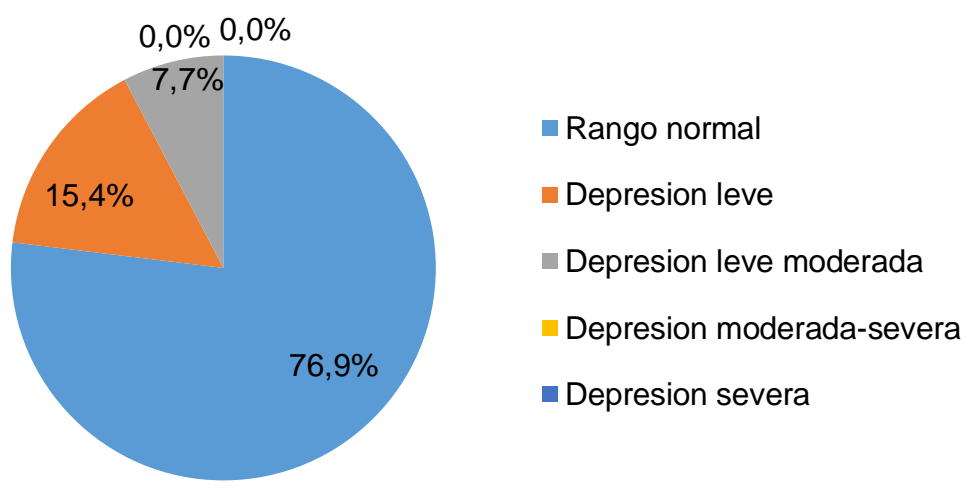

Fig. 2. Nivel de síntomas de depresión al inicio y al final del programa de actividad física para el conjunto de los participantes no cabeza de hogar 
En lo referente a la situación laboral, en el rango de normalidad de depresión en la evaluación inicial se ubicaron el $35 \%$ de quienes se encontraban laborando al momento del estudio y el $50 \%$ de quienes no lo hacían. En la evaluación final en ese rango de normalidad se ubicaron el $75 \%$ de quienes estaban laborando y el $70 \%$ de quienes no laboraban. Para quienes manifestaron ser cabeza de hogar, los resultados indicaron que, en el rango de depresión normal en la evaluación inicial su ubicaron el 38,5\% y el 76,9\% en la evaluación final (Figura 2). Para quienes no eran cabeza de hogar, en ese rango de depresión normal en la evaluación inicial se ubicaron el $41,2 \%$ y en la evaluación final el $70,6 \%$.

\section{Prueba de comparacion de poblaciones relacionadas}

Una vez aplicado el BDII antes y después de los programas de actividad física, se procedió a evaluar el nivel de confiabilidad de los resultados y se encontró que el coeficiente de Alfa de Cronbach fue igual a 0, 83, indicando con ello, una buena confiabilidad de los datos obtenidos. Luego de ello y con el propósito de evaluar si los resulatdos obtenidos antes y despues de los tallers en la escala de depresión de Beck eran significativamente diferentes, se utilizó la prueba de Rangos con Signo de Wilcoxon que es utilizada para este tipo de comparaciones con datos ordinales y dependientes y que no requiere que las muestras se distribuyan de forma normal. Para esto se diseñaron las siguientes hipótesis: i) H0: La mediana de las diferencias entre cada par de datos es mayor de 0,05; y ii) Ha: La mediana de las diferencias de cada par de datos es menor de 0,05 . Los resultados de la aplicación de esta prueba son los que se muestran en la Tabla 2. Dado que el valor de " $p$ " o la significancia asintótica bilateral obtenida de la prueba con Signo de Wilconxon es menor a 0,05 , se rechaza la hipótesis nula, es decir, que los resultados de la aplicación de la escala de depresión de Beck si presentan una diferencia significativa comparada con la aplicación previa a la intervención. En este sentido, la comparación de los resultados antes y después de los talleres de actividad de la escala de depresión de Beck indica que la intervención aplicada tuvo una incidencia positiva en el tratamiento de los síntomas depresivos a nivel general.

Tabla 2. Prueba estadística de Rangos con Signos de Wilconxon.

Abreviatura: beckB: Before (antes) beckA: After (después)

\begin{tabular}{|c|c|}
\hline \multicolumn{2}{|l|}{ Estadísticos de prueba } \\
\hline & beckB - beckA \\
\hline Z & $-3,685^{b}$ \\
\hline Sig. asintótica(bilateral) & 0,000 \\
\hline a. Prueba de Rangos co & \\
\hline
\end{tabular}

\section{DISCUSIÓN}

En general, los resultados del estudio permitieron identificar que la realización organizada de ejercicios físicos de forma frecuente, por parte de los jóvenes universitarios, si permite la reducción de los síntomas depresivos como lo afirman Disease (2015), Guo et al. (2020) y aportan evidencia sobre los efectos positivos que tiene la actividad física en la conducta de las personas como los afirman Hallgren et al. (2020) y Warburton y Bredin (2017) al referirse a las consecuencias positivas de la actividad física regular para la salud mental de la vida en toda persona.

También se pudo confirmar que la actividad física según recomendaciones de los expertos, para que tenga efectos en la salud física y mental se deben realizar de manera moderada o vigorosa para obtener los beneficios esperados y que esta se debe realizar de manera continua de dos a tres veces por semana como lo indica Bailey et al., (2018). De otra parte, aunque el tamaño de la muestra de participantes no es el adecuado para hacer inferencias de la manera diferenciada en que las características sociodemográficas de los participantes como la edad, el estado civil, la presencia de hijos, ser cabeza de hogar, entre otros, influyen en la sintomatología depresiva como lo afirman De los Santos y Carmona (2018) y Currier et al. (2020), los resultados del estudio con mucha limitación si tienden a aportar evidencia en esa dirección.

De otro lado y, teniendo en cuenta que cada día más personas están interesadas en la práctica de actividad física para mejorar no solo la salud sino la calidad de vida, bien sea por recomendación médica o por iniciativa propia, es importante resaltar que tanto la actividad física como el ejercicio propiamente, reducen significativamente los niveles de ansiedad depresión y tristeza y que además trae una serie de beneficios adicionales como la reducción de enfermedades no trasmisibles como las cardiopatías y la obesidad entre otras (Tikanmäki et al., 2017). Es importante señalar que, pese a que existe una gran cantidad de investigaciones al respecto y bastantes aportes desde la literatura sobre el tema, aún existe la necesidad de desarrollar investigaciones que relacionen los beneficios de la actividad física en jóvenes universitarios debido a que algunas de estas investigaciones señalan que los beneficios son indiscutibles y otras indicaron no encontrar dentro de sus resultados, beneficios considerables, pese a que tuvieron en cuenta las recomendaciones de los expertos. 
Vale la pena destacar el hecho de que además de los ejercicios que se realizaron de intensidad moderada en los que se trabajó sobre un rango de 60 a $70 \%$ de la frecuencia cardiaca máxima (FCM) también se desarrollaron actividades centradas en la autoconciencia como el yoga, taichí y stretching, las cuales también aportaron en generar una motivación adicional y que concuerdan con (Whitelaw et al., (2010) al afirmar que estas contribuyen significativamente en la autoimagen, lo que conlleva a mejorar la calidad de vida de los participantes. No obstante, se debe tener en cuenta que este estudio se realizó con personas que no estaban acostumbrados a la actividad física, como tampoco a realizar actividades sobre el 60 y $70 \%$ de su frecuencia cardiaca máxima y que además el estudio se realizó en tiempos de distanciamiento social causado por el Covid -19 y esto pudo ser un factor determinante para los resultados obtenidos y que deben ser considerados para futuras investigaciones.

Finalmente, es importante mencionar que, una gran limitación y debilidad de este estudio, es el no haber contado con un grupo de control que hubiera permitido no solo comparar los resultados de la prueba de síntomas depresivos antes y después del taller de actividad física, sino de los resultados de estudiantes participantes con síntomas que participaron de los talleres con otro grupo de estudiantes también con síntomas que no hubieran participado del taller. Si ello, se hubiera logrado se habría podido tener una gran validez de los resultados, pero desafortunadamente, esto no se pudo hacer para este estudio por el limitado número de estudiantes que decidieron participar. Otra limitación del estudio es la baja representatividad del número de estudiantes, que no permite la generalización de los resultados del estudio por rasgo sociodemográfico avaluado (género, estado civil, rol en la familia, etc.).

\section{CONCLUSIONES}

De acuerdo con los resultados obtenidos en el presente estudio, se puede concluir de manera general que:

(1) Un buen programa de actividad física realizado de manera moderada presenta beneficios en la reducción de los niveles de depresión en jóvenes universitarios debido a que permite generar espacios de bienestar y mejoramiento de la calidad de vida;

(2) Se debe tener en cuenta que en la actualidad la depresión es una patología que afecta a una parte significativa de la población, sobre todo a los jóvenes universitarios por diferentes razones como problemas familiares, académicos y económicos; y

(3) Finalmente, que las prácticas de actividad física basadas en ejercicios de autoconciencia y autoimagen como stretching, yoga y taichí lo cual corrobora lo dicho por Whitelaw (2010), proporcionan un ambiente de tranquilidad y paz interior muy significativos para la reducción de los niveles de depresión en jóvenes universitarios.

\section{AGRADECIMIENTOS}

Los autores del presente artículo agradecen de manera especial a las directivas de las diferentes unidades de las universidades de La Sabana y Minuto de Dios y a los estudiantes que voluntariamente participaron en la investigación, y a quienes apoyaron con su conocimiento y experiencia, por el apoyo prestando de manera continua para el desarrollo del estudio sobre el análisis de los beneficios de la actividad física en jóvenes con síntomas depresivos.

\section{REFERENCIAS}

Bailey, A. P., Hetrick, S. E., y otros tres autores, Treating depression with physical activity in adolescents and young adults: a systematic review and meta-analysis of randomised controlled trials, https://doi.org/10.1017/S0033291717002653, Psychological Medicine, 48(7), 1068-1083 (2018)

Barceló-Soler, A., Baños, R. M., y otros seis autores, Intervenciones de psicoterapia por ordenador para la depresión en Atención Primaria en España, Actas Españolas de Psiquiatría, 47(6), 236-246 (2019)

Bauman, A. E., Reis, R. S., y otros cinco autores, Correlates of physical activity: why are some people physically active and others not?, https://doi.org/10.1016/S0140-6736(12)60735-1, The Lancet, 380(9838), 258-271 (2012)

Biddle, S. J., Pearson, N., y otros dos autores, Tracking of sedentary behaviours of young people: a systematic review, https://doi.org/10.1016/j.ypmed.2010.07.018, Preventive Medicine, 51(5), 345-351 (2010)

Currier, D., Lindner, R., y otros cuatro autores, Physical activity and depression in men: increased activity duration and intensity associated with lower likelihood of current depression. https://doi.org/10.1016/j.jad.2019.09.061, Journal of affective disorders, 260, 426-431 (2020)

Davey, C. G., y McGorry, P. D., Early intervention for depression in young people: a blind spot in mental health care, https://doi.org/10.1016/S2215-0366(18)30292-X, The Lancet Psychiatry, 6(3), 267-272 (2019)

DISEASE, C., Depression in young people often goes undetected, Practitioner, 259(1782), 17-22 (2015) 
De los Santos, P. V., y Carmona Valdés, S. E., Prevalencia de depresión en hombres y mujeres mayores en México y factores de riesgo Población y Salud en Mesoamérica, 15(2), 95-119 (2018)

Guo, F., Tian, Y., y otros cuatro autores, Intensity of physical activity and depressive symptoms in college students: fitness improvement tactics in youth (FITYou) Project doi: 10.2147/PRBM.S267066, Psychology research and behavior management, 13, 787 (2020)

Hallgren, M., Thi-Thuy-Dung, N., y otros cinco autores, Relaciones transversales y prospectivas de las conductas sedentarias pasivas y mentalmente activas y la actividad física con la depresión DOI: 10.1192 / bjp.2019.60, Revista británica de psiquiatría, 217 (2), 413-419 (2020)

Heath, G. W., Parra, D. C., y otros cinco autores, Evidence-based intervention in physical activity: lessons from around the world, https://doi.org/10.1016/S0140-6736(12)60816-2, The Lancet, 380(9838), 272-281 (2012)

Kavanaugh, K., Moore, J. B., y otros dos autores, Correlates of subjectively and objectively measured physical activity in young adolescents, https://doi.org/10.1016/j.jshs.2014.03.015, Journal of Sport and Health Science, 4(3), $222-227$ (2015)

Khouja, J. N., Munafò, M. R., y otros cinco autores, Is screen time associated with anxiety or depression in young people? Results from a UK birth cohort, https://doi.org/10.1186/s12889-018-6321-9, BMC Public Health, 19(1), 1-11 (2019)

Lake, A. A., y Townshend, T. G., Exploring the built environment, physical activity and related behaviours of young people attending school, college and those not in employment, https://doi.org/10.1093/pubmed/fds059, Journal of Public Health, 35(1), 57-66 (2013)

Martínez, M. A. M., de la Luz Martínez Aguilar, M., y cuatro autores, Relación entre obesidad y depresión en adolescentes, https://doi-orgproxyumbeduco/1014198/cuid20185117, Cultura de los Cuidados, 22(51), 154-159 (2018)

McCrorie, P. R., Fenton, C., y Ellaway, A., Combining GPS, GIS, and accelerometry to explore the physical activity and environment relationship in children and young people-a review, https://doi.org/10.1186/s12966-014-0093-

0 , International Journal of Behavioral Nutrition and Physical Activity, 11(1), 1-14 (2014)

Parker, A. G., Hetrick, S. E., y otros cinco autores, The effectiveness of simple psychological and physical activity interventions for high prevalence mental health problems in young people: a factorial randomized controlled trial, https://doi.org/10.1016/j.jad.2016.02.043, Journal of Affective Disorders, 196, 200-209 (2016)

Pascoe, M. C., y Parker, A. G., Physical activity and exercise as a universal depression prevention in young people: anarrative review, https://doi.org/10.1111/eip.12737, Early Intervention in Psychiatry, 13(4), 733-739 (2019)

Pérez-Villalobos, M. V., Cobo-Rendón, R. C., y otros dos autores, Revisión sistemática de la habilidad de autocontrol del estudiante y su rendimiento académico en la vida universitaria http://dx.doi.org/10.4067/S0718-50062018000300049, Formación universitaria, 11(3), 49-62 (2018)

Ruggieri, V., Autismo, depresión y riesgo de suicidio, Revista Medicina, 80, 12-16 (2020)

Sanz Fernández, J., Gutiérrez, S., y otros dos autores, Criterios y baremos para interpretar "el inventario de depresión de Beck-II"(BDI-II), Behavioral Psychology-Psicología Conductual, 22(1), 37-59 (2014)

Sanz, J., y García-Vera, M. P., Rendimiento diagnóstico y estructura factorial del inventario para la depresión de beckSegunda Edición (BDI-II) en pacientes españoles con trastornos psicológicos,

https://doi.org/10.6018/analesps.29.1.130532, Anales de Psicología/Annals of Psychology, 29(1), 66-75 (2013)

Tannehill, D., MacPhail, A., y otros dos autores, What young people say about physical activity: the children's sport participation and physical Activity (CSPPA) study, https://doi.org/10.1080/13573322.2013.784863, Sport, Education and Society, 20(4), 442-462 (2015)

Telama, R., Yang, X., y otros cuatro autores, Physical activity from childhood to adulthood: a 21-year tracking study, https://doi.org/10.1016/j.amepre.2004.12.003, American Journal of Preventive Medicine, 28(3), 267-273 (2005)

Tikanmäki, M., Kaseva, N., y otros cinco autores, Leisure time physical activity in young adults born preterm, https://doi.org/10.1016/j.jpeds.2017.06.068,The Journal of Pediatrics, 189, 135-142 (2017)

Urrego, B. E., Trujillo, D. A., y otros dos autores, Obesidad, depresión, alcoholismo, estrés laboral y nivel de actividad física en una población laboral en el área de la salud de la ciudad de Medellín en el año 2009 (2009)

Van Sluijs, E. M., y Kriemler, S., Reflections on physical activity intervention research in young people-dos, don'ts, and critical thoughts, https://doi.org/10.1186/s12966-016-0348-z, International Journal of Behavioral Nutrition and Physical Activity, 13(1), 25 (2016)

Warburton, D. E., y Bredin, S. S., Health benefits of physical activity: a systematic review of current systematic reviews, https://doi.org/10.1097/HCO.0000000000000437, Current Opinion in Cardiology, 32(5), 541-556 (2017)

Whitelaw, S., Teuton, J., y otros dos autores The physical activity-mental wellbeing association in young people: a case study in dealing with a complex public health topic using a 'realistic evaluation' framework, https://doi.org/10.1016/j.mhpa.2010.06.001, Mental Health and Physical Activity, 3(2), 61-66 (2010)

Zueck, M. D. C., Blanco, L. H., y dos autores. Invarianza factorial de la escala de ansiedad asociada a la imagen corporal en universitarios hombres y mujeres, http://dx.doi.org/10.4067/S0718-50062015000500010, Formación universitaria, 8(5), 91-96 (2015) 